\title{
Limits of Number of Motes at Smart City Scenarios for LoRaWAN
}

\author{
Muammer Catak \\ College of Engineering and Technology, \\ American University of the Middle East, Kuwait \\ Muammer.Catak@aum.edu.kw
}

\author{
Burak Celikkaya \\ Baylan Measuring Instruments \\ Izmir, Turkey \\ b.celikkaya@baylanwatermeter.com
}

\begin{abstract}
LoRaWAN is an emerging technology as a solution to IoT sensor networks. Since it is still in the evolvement stage, academic and industrial researches and applications have to be conducted in order to analyze its limitations and possible improvements. In this paper, the number of motes that could transmit data to a gateway within a $95 \%$ confidence interval has been studied under a number of scenarios. According to the results, the most significant parameter regarding the number of motes is the period of the downlink signal.
\end{abstract}

Keywords-LoRa; LoRaWAN; spreading factor; channel limitations

\section{INTRODUCTION}

Long range wide-area network (LoRaWAN) has an increasing popularity in engineering applications as a lesscomplex wireless communication protocol using the unlicensed spectrum associated with long range (LoRa) modulation [1]. A typical LoRaWAN, consists of a server, a number of gateways and actuators (such as measurement instruments, sensors). An actuator, also called a mote, is connected to a certain gateway via a wireless link while the gateways are connected to the server by IP network means. IoT sensors mostly need to send small amounts of data (uplink) and less frequently receive any data (downlink). Hence, LoRaWAN technology has been employed to transmit data of metering, smart city applications, etc. The key point is to combine low data rate and communication range [2]. LoRaWAN operates in the industrial, science, and medical (ISM) radio bands, and it has to obey to some certain regulations. LoRa can operate in the $868 \mathrm{MHz}$ EU ISM band (Table I), including three compulsory channels at $868.1,868.3$, and $868.5 \mathrm{MHz}$ [3]. Maximum transmission time is bounded by duty cycle percentage in a way that an individual node may only switch its transmitter on for up to 36 seconds in an hour with duty cycle of $1 \%$. The connected nodes are classified into three groups, namely Class A, B, and C. Class A: The mote listens to the Gateway during two short time windows after it has transmitted some data. Therefore, it uses the least power which is ideal for such a battery powered system. Class B: Besides the basic operations of class A, the node can open scheduled windows in order to capture the signals transmitted from the gateway. Class C: The mote listens at all times except from data transmission. Hence, it has to be connected to the mains electricity.
TABLE I. EU ISM BAND POWER AND DUTY CYCLE RESTRICTIONS

\begin{tabular}{|c|c|c|c|}
\hline $\begin{array}{c}\text { Frequency band } \\
\text { (MHz) }\end{array}$ & Applications & $\begin{array}{c}\text { Max. radiated } \\
\text { power (mW-dBm) }\end{array}$ & $\begin{array}{c}\text { Duty } \\
\text { Cycle }\end{array}$ \\
\hline $863.0-864.8$ & IoT & $25-14$ & $0.1 \%$ \\
\hline $864.8-865.0$ & Wireless audio applications & $10-10$ & - \\
\hline $865.0-868.0$ & IoT & $25-14$ & $0.1 \%$ \\
\hline $868.0-868.6$ & IoT & $25-14$ & $1 \%$ \\
\hline $868.6-868.7$ & Alarms & $10-10$ & $1 \%$ \\
\hline $868.7-869.2$ & IoT & $25-14$ & $0.1 \%$ \\
\hline $869.2-869.25$ & Social Alarms & $10-10$ & $0.1 \%$ \\
\hline $869.25-869.3$ & Alarms & $10-10$ & $0.1 \%$ \\
\hline $869.3-869.4$ & Alarms & $10-10$ & $1 \%$ \\
\hline $869.4-869.65$ & IoT & $500-27$ & $10 \%$ \\
\hline $869.65-869.7$ & Alarms & $25-14$ & $10 \%$ \\
\hline $869.7-870.0$ & IoT & & $1 \%$ \\
\hline
\end{tabular}

After an uplink has been transmitted, there are two available receive windows, regarding the downlink messages of Class A. LoRa modulation technique is based on a chirp signal having variable frequency in a well-defined band. The frequency bandwidth of the chirp signal is equivalent to the bandwidth of the data signal. The corresponding data signal is chipped at a higher data rate, and then it is modulated. The chirp signal can be considered as a carrier signal referring to the fundamental modulation theory. The modulation bit rate is defined as [4]:

$$
R_{b}=\frac{S F}{\frac{2^{S F}}{B W}}
$$

where $S F:=$ spreading factor, a number from 7 to 12 , $B W:=$ modulation bandwidth $(125 \mathrm{kHz}$ in this study).

Symbol period and symbol rate, which are reciprocal of the symbol period, are calculated as:

$$
T_{S}=\frac{2^{S F}}{B W} \quad s ; R_{S}=\frac{1}{T_{S}}=\frac{B W}{2^{S F}} \quad \text { symbols } / \mathrm{s}
$$

The chip rate is defined as:

$$
R_{c}=R_{S} 2^{S F}=B W \text { chips } / s
$$

The nominal bit rate of the data signal is:

$$
R_{b}=S F \frac{\frac{4}{4+C R}}{\frac{4}{B W}} \mathrm{bits} / \mathrm{s}
$$


where $C R$ is the code rate, it can be an integer from 1 to 4 . If $(4=4+\mathrm{C} R)$ is defined as rate code, then the nominal bit rate is:

$$
R_{b}=S F \frac{\text { Rate Code }}{\frac{2^{S F}}{B W}} \mathrm{bits} / \mathrm{s}
$$

\section{MATERIALS AND METHODS}

The main objective of this study is to estimate the expected number of motes subject to satisfy a given error band outlined by a described scenario with and without downlink. When the adaptive data rate (ADR) is $\mathrm{ON}$, the spreading factor (SF) of a mote is dynamically reassigned by the gateway regarding to the power of the corresponding received signal. If the signal power is low, most likely the gateway assigns the SF as 12, if the signal power is moderately high enough the SF is most likely assigned as 7. There are 6 levels of SF. If the SF increases the RF sensitivity also increases, which allows signaling propagates longer ranges. On the other hand, the nominal bit rate decreases, which results in increment in time on air (ToA). Detailed information are given in Table II. In this study, the following channels are employed in order to operate the system: 867.1, 867.3, 867.5, 867.7, 867.9, 868.1, 868.3, and $868.5 \mathrm{MHz}$. A class A uplink transmission is followed by two downlink receive windows, during which the motes can capture the data including information from the gateway. The channels are selected randomly and they are assumed to act as uniformly distributed random variables. Since the selections of SF and channel frequencies are completely independent of each other, the joint distribution of SF (assigned as random variable of $\mathrm{X}$ ) and channels (assigned as random variable of $\mathrm{Y}$ ) can be written as:

$$
p_{X Y}(x, y)=p_{X}(x) p_{Y}(y)
$$

TABLE II. SF PROPERTIES

\begin{tabular}{|c|c|c|c|c|c|c|}
\hline$\#$ & SF & $\begin{array}{c}\text { Bandwidth } \\
(\mathbf{k H z})\end{array}$ & $\begin{array}{c}\text { Code } \\
\text { rate }\end{array}$ & $\begin{array}{c}\text { Rate } \\
\text { code }\end{array}$ & $\mathbf{R}_{\mathbf{b}}(\mathbf{b p s})$ & $\begin{array}{c}\text { RF sensitivity } \\
(\mathbf{d B m})\end{array}$ \\
\hline 0 & 12 & 125 & 1 & $4 / 5$ & 293 & -137 \\
\hline 1 & 11 & 125 & 1 & $4 / 5$ & 537 & -136 \\
\hline 2 & 10 & 125 & 1 & $4 / 5$ & 977 & -134 \\
\hline 3 & 9 & 125 & 1 & $4 / 5$ & 1758 & -131 \\
\hline 4 & 8 & 125 & 1 & $4 / 5$ & 3125 & -128 \\
\hline 5 & 7 & 125 & 1 & $4 / 5$ & 5469 & -125 \\
\hline
\end{tabular}

There are 48 options at an instant time, $6(\mathrm{SF}) \times 8($ channels $)$, the uplink signals cannot be overlapped. Therefore, the system can be modeled using 48 individual service stations since the time on air parameters varies at each path. The overlapping probability of two uplink signals can be defined as:

$$
p_{X \mid Y}(x \mid y)=\frac{p_{Y \mid X}(y \mid x) p_{X}(x)}{p_{Y}(y)}
$$

The transmission of uplink packages is a Bernoulli process, since there are merely two possibilities as success or fail [5]. Let the random variable $Z$ be an identically independent Bernoulli distributed with parameter $q \in(0,1)$. The probability of "success" in $N$ trials can be calculated as;

$$
\begin{aligned}
& P(k \mid N \text { trials })=\left(\begin{array}{l}
N \\
k
\end{array}\right) q^{k}(1-q)^{N-k} \\
& =\frac{N !}{(N-k) ! k !} q^{k}(1-q)^{N-k}
\end{aligned}
$$

It is also reported that, a Poisson process can be used as a sufficiently good enough approximation of a Bernoulli process if $N>100, q<0.01$, and $\lambda=N q[6]$ :

$$
e^{-\lambda} \frac{\lambda^{k}}{k !} \cong\left(\begin{array}{l}
N \\
k
\end{array}\right) q^{k}(1-q)^{N-k}
$$

where $k=1,2, \ldots . . \mathrm{N}$.

At the gateway side, the uplink data transmission can be organized as an $\mathrm{M} / \mathrm{D} / \mathrm{c}$ queuing system. The arrivals are governed by Poisson process (denoted as M), the job service time is deterministic, that is the times on air are depending on SF levels (denoted as D). If the gateway is idle at a specific SF and channel combination, then it is assumed that the transmission is successful, otherwise it is considered failed. That means there is no "waiting time" at the gateway side. The vendor reported that if two simultaneous transmissions occur with the same SF and the same channel, the gateway can receive the more powerful signal in the case that the ratio of the powers of the signals is greater than 2 . However, this case is not considered in this study.

\section{RESULTS AND DISCUSSION}

The proposed model has been employed in order to simulate the network with or without downlink signals, since the number of downlink signals has a significant effect on the time-window, during which uplink signals can be transmitted.

TABLE III. TIME ON AIR AND CORRESPONDING FAILURE PROBABILITY OF SFS

\begin{tabular}{|c|c|c|}
\hline SF & ToA (ms) & q $_{\text {SF }}$ \\
\hline 12 & 1710 & $118.75 \times 10^{6}$ \\
\hline 11 & 962.5 & $66.84 \times 10^{6}$ \\
\hline 10 & 468.75 & $32.55 \times 10^{6}$ \\
\hline 9 & 287.5 & $19.97 \times 10^{6}$ \\
\hline 8 & 181.25 & $12.59 \times 10^{6}$ \\
\hline 7 & 125 & $8.68 \times 10^{6}$ \\
\hline
\end{tabular}

\section{A. No Downlink}

The following scenario is employed in order to estimate the number of motes per gateway in an urban residential area. The motes operate in 8 channels with $125 \mathrm{kHz}$ bandwidth. The spreading factors are numbered from 0 (SF 12) to 5 (SF 7). Three different scenarios were employed to assign the probabilities of being in a specific SF level.

\section{1) Scenario $1(\mathrm{S \# 1)}$}

The locations of the motes are classified into three groups: near, middle, and faraway. The number of SF is treated as a random variable governed by Poisson distribution as:

$$
p_{X}(x)=\left\{\begin{array}{c}
\frac{e^{-\lambda \frac{\lambda^{x}}{x !}}}{\sum_{<x>e^{-\lambda} \frac{\lambda^{x}}{x !}}} x=0,1,2, \ldots, N \\
0, \text { otherwise }
\end{array}\right.
$$

The summation term in (10) is necessary for normalization. The parameter is taken as $1 / 6,2 / 8$, and $4 / 8$ for near, middle, and faraway motes respectively. The corresponding probabilities are calculated as $p_{0}=0.12, p_{1}=0.09, p_{2}=$ $0.14, p_{3}=0.16, p_{4}=0.16, p_{5}=0.33$. 


\section{2) Scenario 2 (S\#2)}

The probabilities being in a SF level are assigned as $p_{0}=0.23, p_{1}=0.11, p_{2}=0.08, p_{3}=0.09, p_{4}=0.10, p_{5}=$ 0.39 as suggested by Semtech.

\section{3) Scenario 3 (S\#3)}

The probabilities being in a SF level are $p_{0}=0.28, p_{1}=$ $0.20, p_{2}=0.14, p_{3}=0.10, p_{4}=0.08, p_{5}=0.20$ as reported by [7].

All motes transmit 51-byte frames payload. The time on air of each SF levels and their corresponding failure probabilities are listed in Table III. The total probability of the failure rate up to $k$ failures of $N$ trials can be calculated as:

$$
\sum_{k=1}^{N} \sum_{S F=7}^{12} \frac{e^{-N q_{S F}\left(N q_{S F}\right)^{k}}}{k !}
$$

The results obtained from (11) are listed in Table IV under the case of no downlink for the three different scenarios. Note that the geographic properties of the place, building heights, locations of the meters are not taken into account as parameters of the developed model. Hence, the results obtained from the simulations, can be considered as bounding estimates.

TABLE IV. MAXIMUM NUMBER OF MOTES*

\begin{tabular}{|c|c|c|c|}
\hline \multirow{2}{*}{ Uplink period (h) } & \multicolumn{3}{|c|}{ Number of motes } \\
\cline { 2 - 4 } & S\#1 & S\#2 & S\#3 \\
\hline 1 & 11679 & 9091 & 8164 \\
\hline 4 & 46719 & 36364 & 32655 \\
\hline 6 & 70072 & 54546 & 48983 \\
\hline 8 & 93429 & 72728 & 65310 \\
\hline 16 & 186858 & 145455 & 130619 \\
\hline 24 & 280287 & 218183 & 195929 \\
\hline \multicolumn{4}{|c|}{ *Within 95\% confidence interval without downlink } \\
\hline
\end{tabular}

\section{B. With Downlink}

It assumed that at least 1 downlink occurs within a period of 48 hours per mote. A downlink can be sent by an uplink or it can be sent regardless of the presence of an uplink. There are two options to select the SF of the downlink: i) It could have the same SF if they are 7 or 8 , ii) It is predetermined as SF 9. The expected time on air of a downlink has to be calculated in order to analyze the effects of the downlink signals on the network traffic. Let $Z$ be a random variable representing the time on air of a downlink. According to the total expectation theorem, the unconditional expectation value can be calculated by averaging the conditional expectations:

$$
E[Z]=\sum_{i=1}^{n} E\left[Z \mid A_{i}\right] P\left(A_{i}\right)
$$

The probability of having the same SF with the prior uplink is assigned as $\mu$, then $1-\mu$ is the probability of using a predetermined SF. Hence (12) turns into:

$$
E[Z]=\mu E[Z \mid S F=7 \text { or } 8]+(1-\mu) E[Z \mid S F=9]
$$

The period of 48 hours has been divided into 6 equal 8-hour parts. The probability to have a downlink in the first part is $1 / 6$, and consequently the probability to have no downlink is $5 / 6$. If a downlink transmits, the same probabilities are valid for the second part. If no downlink occurred in the first part, then the probabilities are updated as $1 / 5$, and $4 / 5$ to have a downlink, and to have no downlink, respectively. The entire probability tree is shown in Figure 1.
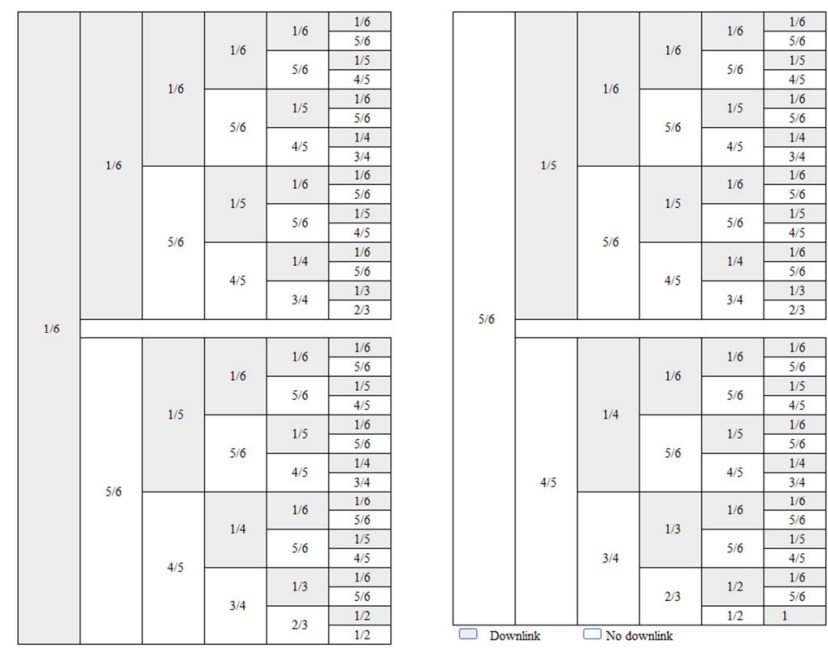

Fig. 1. Possible outcomes of having downlink in $48 \mathrm{~h}$ with $8 \mathrm{~h}$ intervals

The number of downlinks and the corresponding probabilities are given in Table V. At almost $60 \%$, one downlink occurs during the $48 \mathrm{~h}$ period. The general definition for the probabilities can be summarized as:

$$
P(K=k \mid N=n)=\left(\begin{array}{l}
n+1 \\
k+1
\end{array}\right) \frac{k}{n^{k+1}} ;
$$

where $n=6, k=1, \ldots \ldots, 6$

TABLE V. DOWNLINK NUMBER AND CORRESPONDING PROBABILITIES

\begin{tabular}{|c|c|}
\hline \# of Downlink & Probability \\
\hline 1 & $21 / 36$ \\
\hline 2 & $70 / 216$ \\
\hline 3 & $105 / 6^{4}$ \\
\hline 4 & $84 / 6^{5}$ \\
\hline 5 & $35 / 6^{6}$ \\
\hline 6 & $6 / 6^{7}$ \\
\hline
\end{tabular}

The number of motes with the case of at least one downlink transmitted during the $48 \mathrm{~h}$ period at various scenarios is shown in Table VI. Comparing with the results shown in Table IV, the downlink transmission dramatically affects the number of motes that a gateway can handle within an acceptable error band.

TABLE VI. MAXIMUM NUMBER OF MOTES*

\begin{tabular}{|c|c|c|c|}
\hline \multirow{2}{*}{ Uplink period (h) } & \multicolumn{3}{|c|}{ Number of motes } \\
\cline { 2 - 4 } & S\#1 & S\#2 & S\#3 \\
\hline 1 & 552 & 552 & 533 \\
\hline 4 & 1108 & 1251 & 1074 \\
\hline 6 & 1358 & 1489 & 1316 \\
\hline 8 & 1569 & 1692 & 1521 \\
\hline 16 & 2221 & 2327 & 2154 \\
\hline 24 & 2721 & 2820 & 2640 \\
\hline \multicolumn{4}{|c|}{ *Within 95\% confidence interval with downlink }
\end{tabular}


The expected number of downlink is calculated 1.5216 as:

$$
E[W]=\sum_{w} p_{W}(w)
$$

where $w$ and the corresponding probabilities $p_{w}(w)$ are supplied in Table VI. With the same manner, the expected ToAs are calculated for S\#1, S\#2, and $\mathrm{S} \# 3$ as 252.27, 250.55, and $267.04 \mathrm{~ms}$, respectively.

\section{CONCLUSION}

The number of motes that can be handled by a gateway using LoRaWAN technology within 95\% confidence interval has been studied. Various scenarios have been employed to a smart city application. Under the condition of no downlink transmission, a gateway can easily company a huge number of motes. However, with downlink transmission, the number of motes transmitting data with $95 \%$ confidence interval decreases dramatically. The possible solutions to overcome this effect could be the increase of the period of uplink and/or downlink, or the increase of the number of gateways, but both solutions increase the application cost. In the future, appropriate location estimation of a gateway in order to increase the network quality will be carried out.

\section{REFERENCES}

[1] K. Mikhaylov, J. Petajajarvi, J. Janhunen, “On lorawan scalability: Empirical evaluation of susceptibility to inter-network interference", European Conference on Networking and Communications, Oulu, Finland, June 12-15, 2017

[2] F. Adelantado, X. Vilajosana, P. Tuset-Peiro, B. Martinez, J. MeliaSegui, T. Watteyne, "Understanding the limits of LoRaWAN", IEEE Communications Magazine, Vol. 55, No. 9, pp. 34-40, 2017

[3] D. P. Bertsekas, J. N. Tsitsiklis, Introduction to Probability, Athena Scientific, 2002

[4] Semtech, LRra Modulation Basics, AN1200.22, 2015

[5] B. Vejlgaard, M. Lauridsen, H. Nguyen, I. Z. Kovacs, P. Mogensen, M. Sorensen, "Interference impact on coverage and capacity for low power wide area iot networks", IEEE Wireless Communications and Networking Conference, San Fransisco, USA, March 19-22, 2017

[6] ETSI, ETSI 300 220-1 (v2. 4.1), Electromagnetic Compatibility and Radio Spectrum Matters (ERM), 2012

[7] D. Bankov, E. Khorov, A. Lyakhov, "On the limits of lorawan channel access", International Conference on Engineering and Telecommunication, Moscow, Russia, November 29-30, 2016 